\title{
Stachydrine Protects Against Pressure Overload-Induced Cardiac Hypertrophy by Suppressing Autophagy
}

\author{
Tong-Tong Cao ${ }^{a}$ Hui-Hua Chen ${ }^{a}$ Zhiwei Dong ${ }^{b}$ Yan-Wu Xuc Pei Zhao ${ }^{d}$ Wei Guo ${ }^{a}$ \\ Hong-Chang Wei ${ }^{a}$ Chen Zhang ${ }^{\text {a }}$ Rong Lu ${ }^{\mathrm{a}}$
}

\begin{abstract}
aDepartment of Pathology, Shanghai University of Traditional Chinese Medicine, Shanghai, 'bnstitute of Burn Research, Southwest Hospital, State Key Laboratory of Trauma, Burns and Combined Injury, Third Military Medical University, Chongqing, 'Department of Biochemistry, Shanghai University of Traditional Chinese Medicine, Shanghai, dExperiment Center, Shanghai University of Traditional Chinese Medicine, Shanghai, China
\end{abstract}

\section{Key Words}

Stachydrine • Cardiac hypertrophy • Autophagy • ROS • NOX2

\begin{abstract}
Background: Autophagy is required for the maintenance of cardiomyocyte homeostasis. However, excessive autophagy plays a maladaptive role in pressure overload-induced heart failure. To identify mechanisms by which Stachydrine inhibits pressure overload-induced cardiac hypertrophy, we determined inhibitory activities against activation of NADPH oxidase, reactive oxygen species(ROS) production and excessive activation of autophagy. Methods: Stachydrine was administered intragastrically to Wistar rats after Transverse aortic constriction(TAC) and $\mathrm{H} 9 \mathrm{c} 2$ cells were treated with Stachydrine after Angiotension II stimulation. The activation of NADPH oxidase2 required the membrane translocation of p47phox and p67phox. Cell membrane fraction was isolated by ultracentrifuge in sucrose. The expression of p67phox, p47phox, gp91phox subunit in the cell membrane were determined by western blot. The combination of p67phox and gp91 phox subunit was detected by immunofluorescence staining. The expression of phosphorylated p47phox subunit was determined by western blot. The intracellular ROS were measured with DCF-DA fluoresence. The autophagic flux was measured by recording the fluorescence emission of the fusion protein mRFP-GFP-LC3 by dynamic live-cell imaging. Reuslts: We report here that stachydrine, a major constituent of Leonurus heterophyllus Sweet, inhibited AngII-induced excessive autophagy within H9c2 cells. Stachydrine blocked the over phosphorylation of the p47phox subunit, decreased the translocation of p47phox and p67phox to the membrane, inhibited the activity of NOX2, and reduced the generation of ROS. We also demonstrated that stachydrine ameliorated TACinduced cardiac hypertrophy, dysfunction and excessive autophagy in vivo. Conclusions: Our study highlights the importance of regulating NOX2 when autophagy is obviously activated. By inhibiting NOX2, Stachydrine inhibits ROS production, thus exerting a remarkable activity of inhibiting hypertrophy, which could have considerable effect on clinical practice.
\end{abstract}

T.-T. Cao, H.-H. Chen and Z. Dong contributed equally to this work.

(C) 2017 The Author(s)

Published by S. Karger AG, Base

Chen Zhang or Rong Lu

Department of Pathology, Shanghai University of Traditional Chinese Medicine, Shanghai,

No.1200 Cailun Road, Shanghai, 201203, (China)

E-Mail zhangchen@shutcm.edu.cn / lurong@shutcm.edu.cn) 


\section{Cellular Physiology \begin{tabular}{ll|l} 
and Biochemistry $10.1159 / 000477119$ & $\begin{array}{l}\text { Do } 2017 \text { The Author(s). Published by S. Karger AG, Basel } \\
\text { Pww.karger.com/cpb }\end{array}$ \\
\hline
\end{tabular} \\ Cao et al.: Stachydrine Protects Against Cardiac Hypertrophy by Suppressing Autophagy}

\section{Introduction}

In response to stress from hypertension, neurohumoral overactivation, or other myocardial injury, the heart initially compensates with an adaptive hypertrophic increase in mass. However, sustained or excessive hypertrophic responses may lead to a transition from compensated hypertrophy to heart failure [1]. Cardiac remodeling involves alterations in the balance between protein synthesis and protein degradation. In recent years, the importance of autophagy in the development of heart failure has been documented. Autophagy is a highly conserved catabolic cellular process for protein and nutrient recycling via a lysosomalmediated pathway. Excessive and chronic autophagy has been reported to be able to lead to excessive degradation of critical cellular proteins and organelles, even leading to cell death, and plays a maladaptive role in pressure overload-induced cardiac remodeling and heart failure [2]. Thus, modulating autophagy provides an attractive potential therapeutic target for treating heart failure.

The renin-angiotensin system (RAS), especially angiotensin II (AngII), has been shown to play a major role in heart failure. Many of the downstream effects of AngII signaling are mediated by reactive oxygen species (ROS) and oxidative stress. NADPH oxidase (NOX) family proteins are devoted to the dedicated generation of ROS. Recent studies have demonstrated that NOX-derived ROS were among the main intracellular signal transducers for sustaining autophagy [3].

Leonurus heterophyllus Sweet, commonly called motherwort, is a popular herbal medicine that has been used in China for centuries. During the last few decades, experimental and clinical studies have demonstrated the cardiovascular beneficial effects of motherwort [4]. Recently, our group demonstrated that stachydrine, one of the major constituents of Leonurus heterophyllus Sweet, attenuates norepinephrine-induced cardiomyocyte hypertrophy [5]. Our group also found that stachydrine attenuated AngII-induced cardiac myocyte hypertrophy and the excessive production of ROS [6].

Here, we report that stachydrine inhibited the excessive autophagy activity within H9c2 cells induced by AngII and in a rat model subjected to the TAC operation. We found that stachydrine abrogated the enhanced NOX2 activity and ROS generation induced by AngII and ameliorated pressure overload-induced cardiac hypertrophy and heart dysfunction.

\section{Materials and Methods}

\section{Cell Culture and Treatment}

The H9c2 cell line was purchased from Shanghai Institutes for Biological Science and cultured in Dulbecco's Modified Eagle's Medium (DMEM, Gibco BRL, Grand Island, NY, USA) containing 10\% fetal bovine serum (FBS), 100 units $/ \mathrm{ml}$ of penicillin, and $100 \mu \mathrm{g} / \mathrm{ml}$ of streptomycin at $37^{\circ} \mathrm{C}$ in a humidified incubator with $5 \% \mathrm{CO}_{2}$. After the cells were incubated for $24 \mathrm{~h}$ and attached to the plate, they were left untreated or were treated with $1 \mu \mathrm{M}$ AngII in the presence or absence of $1 \mu \mathrm{M}$ telmisartan or $1 \mu \mathrm{M}$ stachydrine for $24 \mathrm{~h}$. To regulate autophagy, 3-methyladenine (3-MA, $5 \mathrm{mM}$ ) or rapamycin $(0.5 \mathrm{mM})$ was added for $2 \mathrm{~h}$ before the addition of AngII and incubation for $24 \mathrm{~h}$. After treatment, the cells were harvested for analysis.

\section{mRFP-GFP-LC3 Assay}

Autophagic flux was measured as described previously [20]. Briefly H9c2 cells were plated on the coverslips and incubated with an mRFP-GFP-LC3 lentivirus (MOI 15, Hanbio. Co. LTD, Shanghai, China). After $36 \mathrm{~h}$ of infection, confocal images were obtained at 561 and $488 \mathrm{~nm}$ using a $63 \times$ oil immersion objective (iXon Ultra 897 CCD). The puncta of each cell were counted, and 10 pictures were taken for each sample. The GFP signal is sensitive to the acidic conditions of the lysosome lumen, whereas mRFP is more stable. Therefore, colocalization of both GFP and mRFP fluorescence indicates a compartment that has not fused with a lysosome. In contrast, an mRFP signal without GFP indicates a compartment fused with a lysosome. So, autophagy flux was then measured by confocal counting of GFP+/mRFP+(yellow) and GFP-/mRFP+(red) puncta [7]. 


\section{Cellular Physiology Cell Physiol Biochem 2017;42:103-114 \\ \begin{tabular}{ll|l} 
and Biochemistry Published online: May 12, 2017 & $\begin{array}{l}\text { C } 2017 \text { The Author(s). Published by S. Karger AG, Basel } \\
\text { www.karger.com/cpb }\end{array}$ \\
\hline
\end{tabular} \\ Cao et al.: Stachydrine Protects Against Cardiac Hypertrophy by Suppressing Autophagy}

\section{Measurements of ROS}

To evaluate ROS generation, we used a modified method as described previously[8]. Briefly, cells were cultured in glass-bottom dishes and loaded with $2.5 \mu \mathrm{mol} / \mathrm{L}$ of H2DCFDA (Thermo fisher scientific, Waltham, MA, USA) for $20 \mathrm{~min}$. The dishes were then mounted on a Carl Zeiss Axio Observer Z1 microscope with a Sutter Lambda DG-4 monochromator and filter sets. Cells were illuminated at a very low light intensity of $488 \mathrm{~nm}$, and pictures were taken at 10 frames/s using a $40 \times$ oil immersion objective at $510 \mathrm{~nm}$ with an iXon Ultra 897 CCD. The rates of change of the DCF fluorescence signal within a single cell were calculated to report the rate of ROS generation. This method can avoid the difference in DCF loading and resting signal between cells.

\section{Experimental Animals and TAC Operation}

All animal procedures described in this study were approved by the Animal Care and Use Committee of Shanghai University of Traditional Chinese Medicine. Male Wistar rats (3 months old, weighing 145-155 g) were purchased from the Shanghai Laboratory Animal Center (Shanghai, China). The animals were housed at room temperature under a $12 \mathrm{~h}$ light/dark cycle with free access to water and a standard diet. The transverse aorta constriction (TAC) surgery was used to generate pressure overload-induced cardiac hypertrophy and heart failure. Briefly, rats were anesthetized with isoflurane (Inspira-ASV, Harvard Apparatus, Holliston, MA, USA) and placed supine on a warm electric pad (World Precision Instruments, Inc. Sarasota, FL, USA). The transverse aortic arch was ligated between the innominate artery and the left carotid artery with an 18-gauge intravenous catheter using a 4-0 nylon suture. The catheter was removed after the ligation. Rats in the sham group underwent all operation procedures except for the ligation. Animals subjected to TAC were treated with telmisartan $(5 \mathrm{mg} / \mathrm{kg})$, stachydrine $(8 \mathrm{mg} / \mathrm{kg})$, or vehicle control by oral gavage for 12 weeks.

\section{Echocardiography Analysis}

The rats were anesthetized with $2.5 \%$ isoflurane in 95\% oxygen and 5\% carbon dioxide and then situated in the supine position on a warming platform to maintain the core temperature at $37^{\circ} \mathrm{C}$. Cardiac function was evaluated by echocardiography using a high-resolution small animal imaging system (Vevo2100, VisualSonics Inc, Toronto, Canada). Two-dimensional and M-mode echocardiographic images of the long and short axis were recorded. On the two-dimensional recordings of the short axis at the midpapillary muscle level, the left ventricular anterior wall thickness, posterior wall thickness, interventricular septal wall thickness in diastole and systole, and left ventricular end-diastolic and end-systolic diameters were measured.

\section{Hemodynamic Measurements}

Animals were anesthetized with isoflurane, fixed, intubated and respirated. After the chest was opened, a 1.9F pressure-volume (PV) conductance catheter (Scisence, Ontario, Canada) was inserted into the left ventricle via an apex approach. Pressure-volume data were recorded and analyzed using LabScribe 2 software (iWorx, Dover, NH, USA). Tracings of the left ventricular pressure and volume were collected at a sampling rate of $1000 \mathrm{~Hz}$. After stabilization for $15 \mathrm{~min}$, data were recorded at steady-state baseline conditions and during transient inferior vena cava occlusions.

\section{Histology and Immunohistochemistry}

All myocardium tissues were fixed in $4 \%$ paraformaldehyde overnight at $4^{\circ} \mathrm{C}$, rinsed, and transferred to PBS followed by paraffin embedding. Sections were stained with hematoxylin and eosin. For the immunohistochemistry assay, after deparaffinization, the sections (4-mm thick) were incubated with a primary antibody against microtubule-associated protein-1 light chain 3 (LC3, Cell Signaling Technology, Danvers, MA, USA), followed by Alexa Fluor 488 (green, Thermo fisher scientific, Waltham, MA, USA). Sections were also labeled with an anti-cTNT(cardiacspecific troponin T) antibody (Abcam, Cambridge, CB, UK) followed by Alexa Fluor 568 (red, fisher scientific, Waltham, MA, USA).

\section{Electron Microscopy}

Cardiac tissue was rapidly cut into 1-mm cubes, immersion fixed in $2.5 \%$ glutaraldehyde in $0.1 \mathrm{M}$ phosphate buffer (pH 7.4) overnight at $4^{\circ} \mathrm{C}$. After fixation, the selections were immersed in $1 \%$ buffered osmium tetroxide for $2 \mathrm{~h}$. The specimens were then dehydrated through a graded ethanol series and 


\section{Cellular Physiology Cell Physiol Biochem 2017;42:103-114

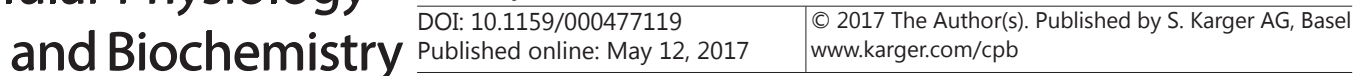 \\ Cao et al.: Stachydrine Protects Against Cardiac Hypertrophy by Suppressing Autophagy}

embedded in epoxy resin. After that, the selections were incised into ultrathin sections $(60-70 \mathrm{~nm})$ with an ultramicrotome and post-stained with uranyl acetate and lead citrate. Then, sections were examined under an H-800 electron microscope (Hitachi, Tokyo, Japan).

\section{Preparation of the Membrane Fraction}

Cells were lysed in buffer containing $250 \mathrm{mM}$ sucrose, $20 \mathrm{mM}$ HEPES (7.4), $10 \mathrm{mM} \mathrm{KCl}, 1.5 \mathrm{mM} \mathrm{MgCl}$, $1 \mathrm{mM}$ EDTA(Ethylenediaminetetraacetic acid), and $1 \mathrm{mM}$ DTT(1,4-Dithiothreitol). The lysate was separated by centrifugation at 100,000 $\mathrm{g}$ for $1 \mathrm{~h}$. The membrane fraction was obtained by suspending the pellet with lysis buffer.

\section{Western Blot Analysis}

Protein lysates from H9c2 cells, heart tissue, or membrane fractions were loaded and separated by $12 \%$ SDS-PAGE and then transferred to PVDF membranes (EMD Millipore, MA, USA). The membranes were probed overnight with primary antibodies at $4^{\circ} \mathrm{C}$. The primary antibodies were anti-LC3 (Cell Signaling Technology, Danvers, MA, USA), anti-p62 (Abcam, Cambridge, CB, UK), anti-p47phox (Abcam, Cambridge, CB, UK), anti-Phospho-p47phox (Ser304) (Abcam, Cambridge, CB, UK), anti-p67phox (Abcam, Cambridge, CB, UK), anti-gp91phox (Abcam, Cambridge, CB, UK), anti-mTOR (Cell Signaling Technology, Danvers, MA, USA), anti-Phospho-mTOR (Ser2448, Cell Signaling Technology, Danvers, MA, USA), anti-AMPK(Cell Signaling Technology, Danvers, MA, USA), anti-phospho-AMPK (Thr172, Cell Signaling Technology, Danvers, MA, USA), anti- $\beta$-actin (Cell Signaling Technology, Danvers, MA, USA), and anti- $\alpha 1$ subunit of $\mathrm{Na}^{+} / \mathrm{K}^{+}$-ATPase ( $\alpha 1 \mathrm{ATP} 1$, Abcam, Cambridge, CB, UK). Membranes were incubated with the appropriate secondary antibodies for $1 \mathrm{~h}$ at room temperature. The signals were detected using Immobilon Western Chemiluminescent HRP Substrate (EMD Millipore, MA, USA) and quantified by densitometry using an Image Analysis System. The data were normalized by $\beta$-actin for whole-cell lysates or by $\alpha 1$ ATP1 for membrane fractions to ensure equal loading and were expressed as a ratio of the experimental groups to the control group.

\section{Statistical Analysis}

Results are presented as the means \pm SEM. The data were analyzed with 1-way ANOVA, and group differences were detected using a Bonferroni post hoc test when the initial ANOVA analysis revealed statistically significant differences. $P$ values less than 0.05 were considered significant.

\section{Results}

Stachydrine inhibited AngII-induced excessive autophagy in H9c2 cells

Recent studies have suggested a maladaptive role of autophagy in pressure overloadinduced cardiac dysfunction. To determine whether stachydrine can block the excessive autophagy induced by AngII in H9c2 cells, we evaluated the LC3II/LC3I ratio by western blotting. As shown in Fig. 1B, the LC3II/LC3I ratio was dramatically increased by AngII treatment. Stachydrine significantly blocked this increase. Then, we used H9c2 cells transfected with RFP-GFP-LC3 adenovirus to further investigate which step of autophagic flux was affected by stachydrine. GFP is sensitive to the acidic $\mathrm{pH}$ inside the lysosome and will lose fluoresce once an autophagosome fuses with the lysosome to form an autolysosome. However, RFP is resistant to the low PH, and these autolysosomes now stain as red only. In green/red merged images, yellow puncta (RFP+/GFP+) indicate autophagosomes, while red puncta (RFP+/GFP-) indicate autolysosomes (As shown in Fig. 1C). Statistical analysis indicates that stachydrine inhibited the AngII-induced enhancement of both autophagosomes (yellow puncta) and autolysosomes (red puncta). Next, we evaluated the phosphorylation levels of MTOR and AMPK as cell signaling markers of autophagy in the H9c2 cells. Mammalian target of rapamycin (mTOR) is a serine/threonine kinase that integrates growth factors and nutrient signals to inhibit autophagy and promote cell growth. Another protein involved in autophagy regulation is AMPK. AMPK is phosphorylated, leading to its activation and autophagy induction [9]. As shown in Fig. 1E and 1F, AngII suppressed the phosphorylation level of mTOR at Ser2448 and promoted the phosphorylation level of 
A<smiles>C[N+]1(C)CCCC1C(=O)O</smiles>

C

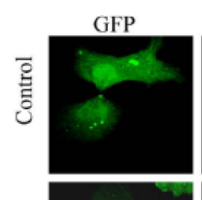

$\underset{\substack{\bar{y} \\ \dot{z}}}{=}$
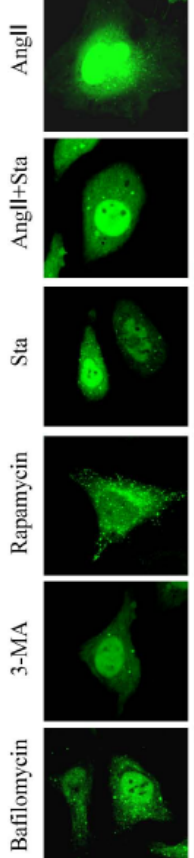

B
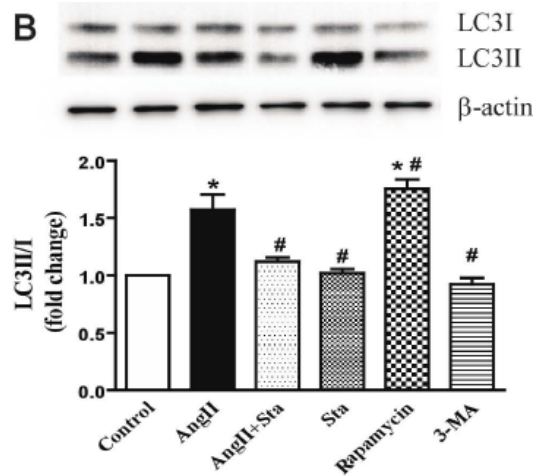

D

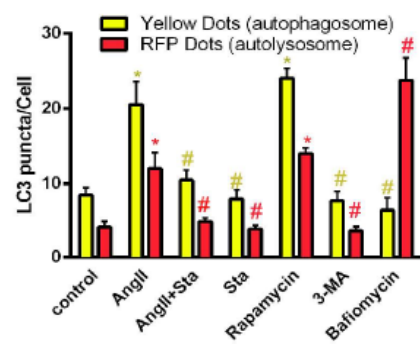

E
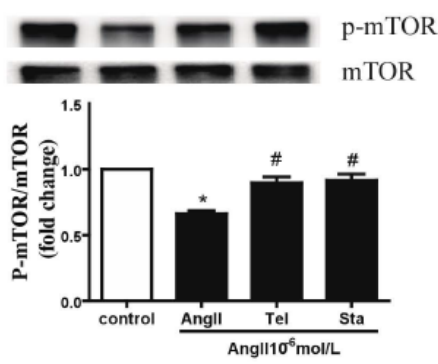

$\mathrm{F}$
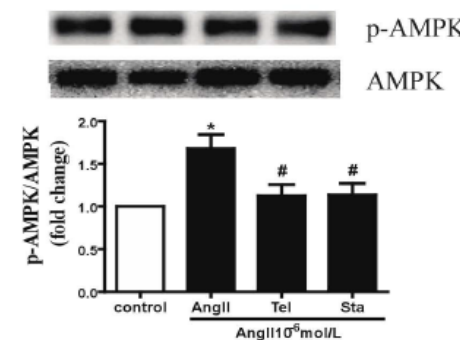

Fig. 1. Stachydrine inhibited AngII-induced excessive autophagy in H9c2 cells. (A) Chemical structure of stachydrine. (B) SDS-PAGE and western blotting analysis of LC3I and LC3II on H9c2 cell lysates and the quantification of the ratio of LC3II/LC3I. (C) Representative confocal fluorescent images of H9c2 cells with the tandem fluorescent-tagged LC3 assay, $\times 400$. (D) Quantification of the number of autophagosome (GFP+/ $\mathrm{RFP}+$,yellow) and autolysosome (GFP-/RFP+, red) puncta in H9c2 cells. (E) SDS-PAGE and western blotting analysis of phosphorylation of mTOR at Ser2448. (F) SDS-PAGE and western blotting analysis of phosphorylation of AMPK at Thr172. The data are expressed as the mean \pm SEM. ${ }^{*} \mathrm{P}<0.05$ compared to control; \# $\mathrm{P}<0.05$ compared to AngII. 3-MA, 3-methyladenine; AngII, angiotensin II; Sta, stachydrine; Tel, telmisartan.

AMPK at Thr172. Stachydrine almost completely prevented those changes. Taken together, our data show that stachydrine decreased the excessive autophagic activity induced by AngII in $\mathrm{H} 9 \mathrm{c} 2$ cells. 


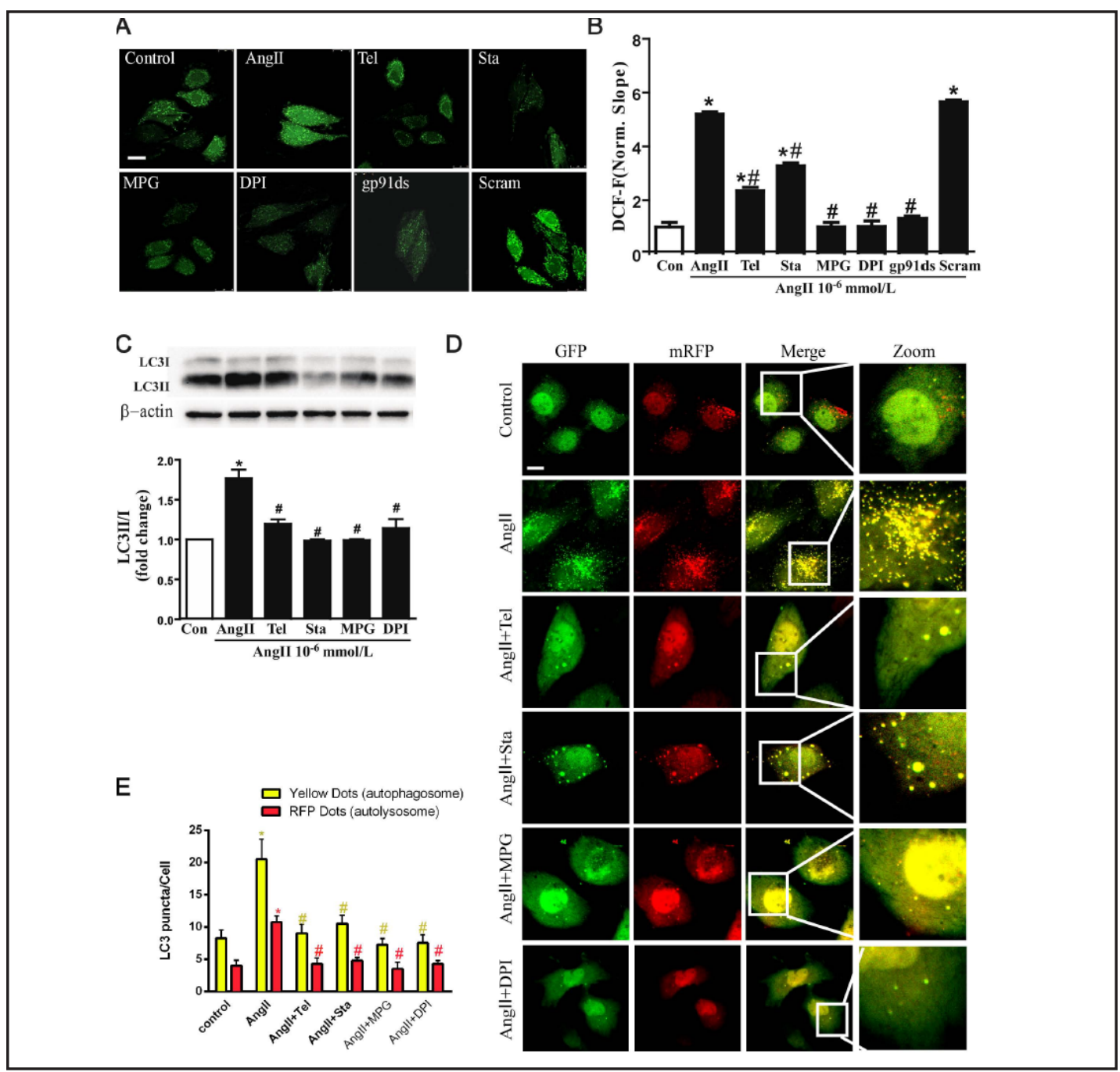

Fig. 2. Stachydrine restored AngII-induced ROS over-generation in H9c2 cells. (A) Representative DCF fluorescent images, $\times 400$. (B) Quantification of the slope of the DCF fluorescent signal. (C) SDS-PAGE and western blotting analysis of the ratio of LC3II/I. (D) Representative confocal fluorescent images of H9c2 cells with the tandem fluorescent-tagged LC3 assay, $\times 400$. (E) Quantification of the number of autophagosome $(\mathrm{GFP}+/ \mathrm{RFP}+$, yellow) and autolysosome (GFP-/RFP+, red) puncta in H9c2 cells. The data are expressed as the mean \pm SEM. * P $<0.05$ compared to control; \# P<0.05 compared to AngII. AngII, angiotensin II; DPI, diphenyleneiodonium; MPG, N-(2-mercaptopropionyl) glycine; Sta, stachydrine; Tel, telmisartan.

\section{Stachydrine restored AngII-induced ROS over-generation in H9c2 cells}

Many of the downstream effects of AngII signaling are mediated by elevated levels of reactive oxygen species (ROS) produced by NADPH oxidase (NOX) [10]. To test whether stachydrine has the potential to the reduce ROS generation caused by AngII, we measured ROS generation with 2', 7'-dichlorofluorescein diacetate (DCF). The changing rate of the DCF fluorescence signal indicates the generation rate of ROS within the cells. As shown in Fig. 2B, the presence of stachydrine, antioxidant MPG, the NOX inhibitor DPI (diphenyleneiodonium) or the NOX2-specific inhibitor gp91ds-tat blocked the AngII-induced elevation in ROS production in H9c2 cells. Moreover, stachydrine as well as MPG, DPI, and gp91ds-tat blocked the increase in autophagic activity induced by AngII (Fig. 2C, 2D, 2E). These data suggest that stachydrine might inhibit AngII-induced excessive autophagic activity by blocking ROS production. 


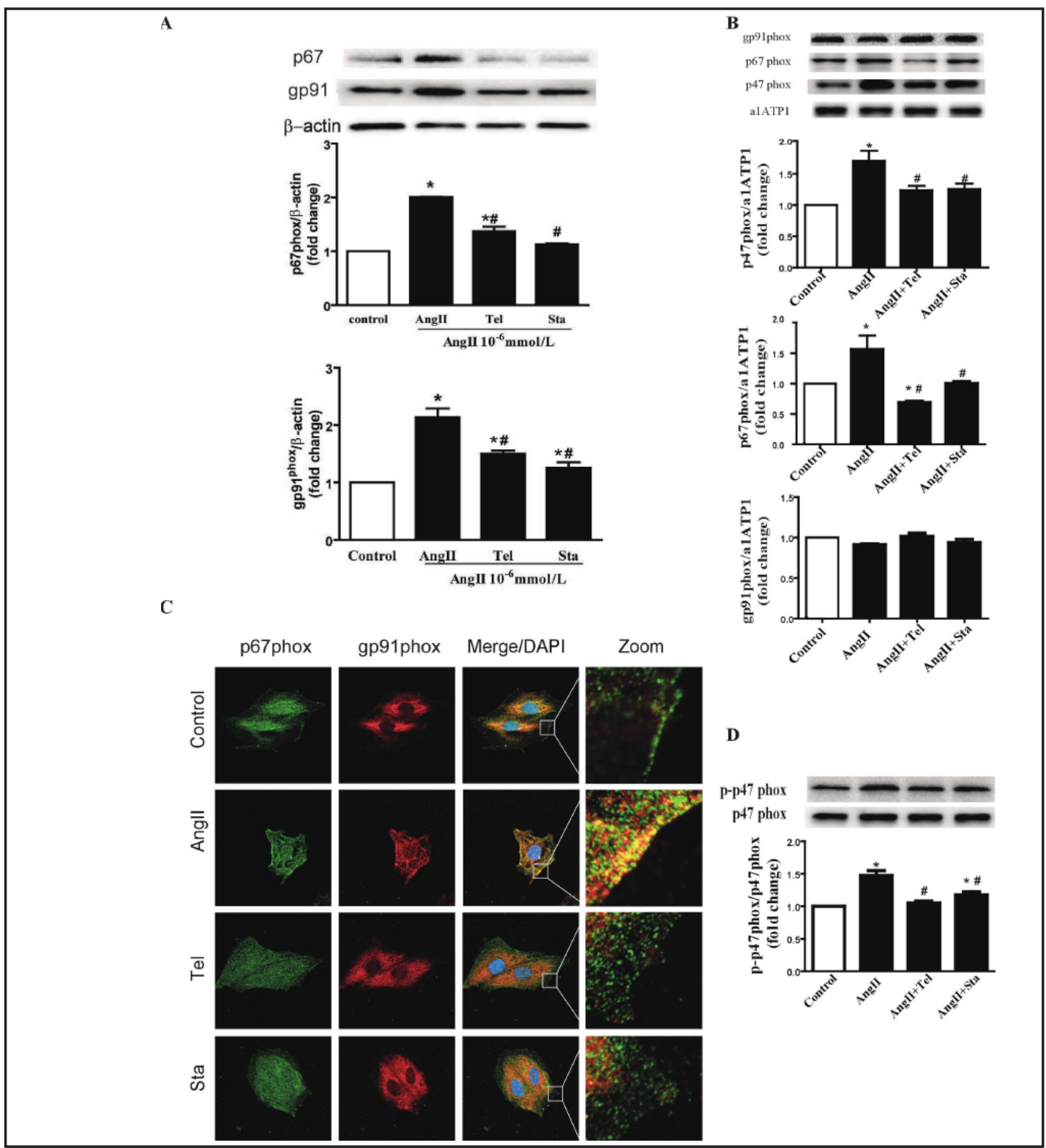

Fig. 3. Stachydrine inhibited AngII-induced NOX2 over-activation in H9c2 cells. (A) SDS-PAGE and western blotting analysis of the expression of gp91phox and gp67phox from total H9c2 cell lysates. (B) SDS-PAGE and western blotting analysis of gp91phox, p47phox and p67phox from the membrane fraction of H9c2 cells. (C) Representative pictures of the co-localization of gp91phox and p47phox; p47phox was visualized with the Alexa Fluor 488-conjugated stain (green), whereas gp91phox was stained with Alexa Fluor 568 (red), $\times 400$. (D) SDS-PAGE and western blotting analysis of phosphorylation of p47phox at Ser345. The data are expressed as the mean \pm SEM. ${ }^{*} \mathrm{P}<0.05$ compared to control; \# $\mathrm{P}<0.05$ compared to AngII. AngII, angiotensin II; Sta, stachydrine; Tel, telmisartan.

Stachydrine inhibited AngII-induced NOX2 over-activation in H9c2 cells

NOX2 and NOX4 are the most abundantly expressed isoforms of NOX in cardiomyocytes. A significant body of evidence indicates that NOX2 is involved in mediating signal transduction of AngII [3]. This is consistent with our results that gp91ds-tat blocked the AngII-induced increase in ROS generation (Fig. 2B). Therefore, we asked whether stachydrine can regulate ROS production by inhibiting the activity of NOX2. To address this question, we evaluated the expression of gp91phox and p67phox in the whole cells by western blot. As shown in Fig. 
A

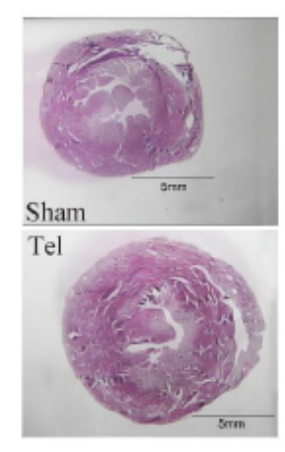

B
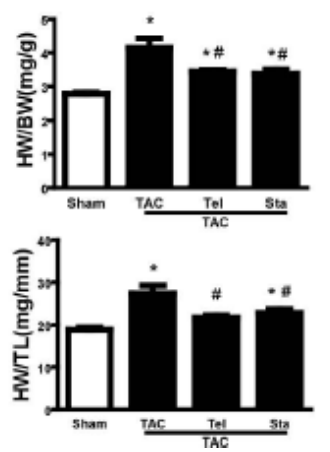

\section{C}

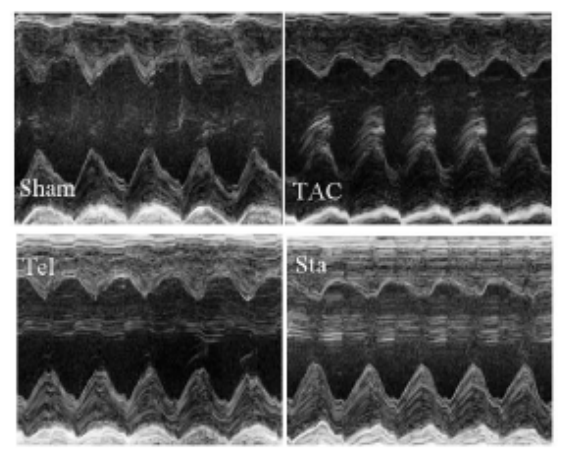

D
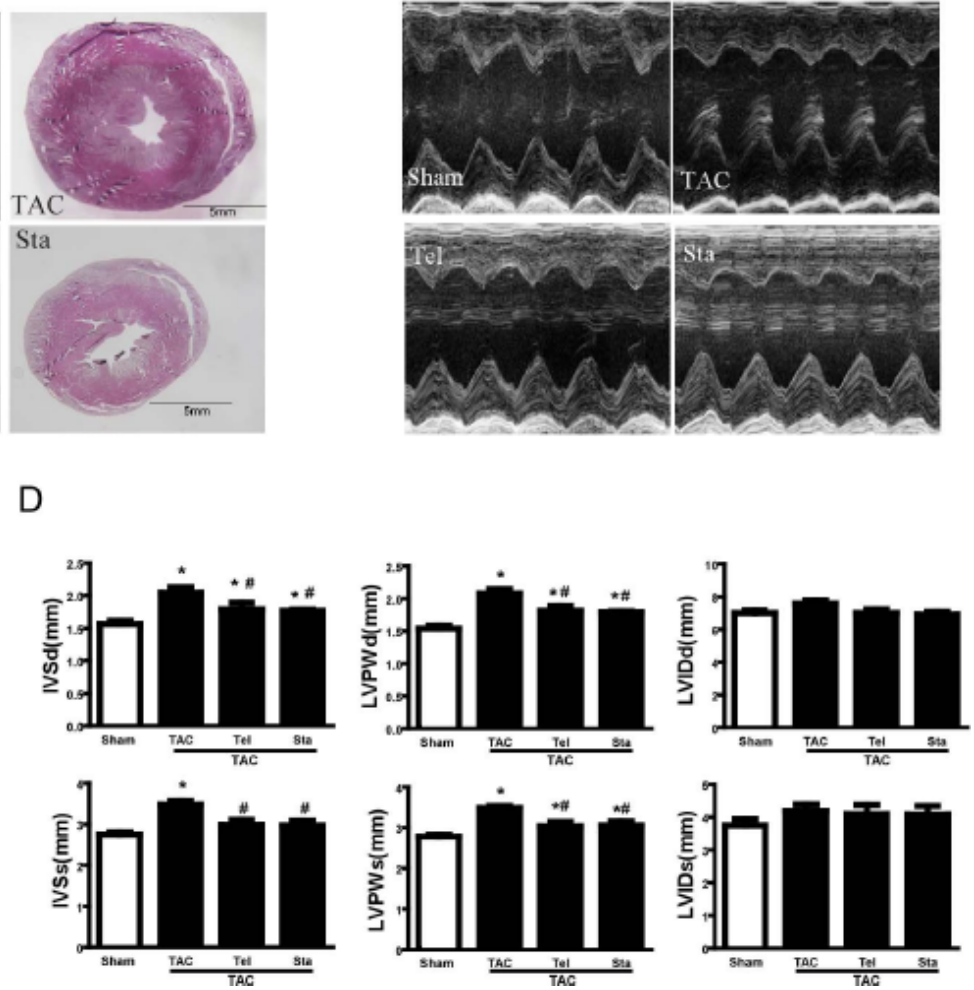

Fig. 4. Stachydrine alleviated TAC-induced cardiac hypertrophy in rats. (A) Cardiac images; hearts were sectioned at the papillary muscle level, $\times 8$. (B) Quantification of the heart weight-to-body weight ratio and heart weight-to-tibial length ratio. (C) Representative M-mode echocardiography tracings. (D) Quantification of the echocardiographic parameters. The data are expressed as the mean \pm SEM. ${ }^{*} \mathrm{P}<0.05$ compared to Sham; \# P<0.05 compared to TAC. Sta, stachydrine; TAC, transverse aorta constriction; Tel, telmisartan.

3A, AngII induced a remarkable increase in the expression of gp91phox and p67phox, which was abrogated by stachydrine. In addition to the protein expression, the activity of NOX2 is largely dependent on the translocation of cytosolic subunits. Membrane-bound gp91phox requires association with cytosolic regulatory subunits, including $\mathrm{p} 47 \mathrm{phox}$ and p67phox, to produce ROS. Therefore, we examined the localization of the cytosolic subunits of NOX2. As shown in Fig. 3B, the amount of p47phox and p67phox within the membrane fraction was significantly increased by AngII and was restored to normal levels by stachydrine. These data were supported by the immunofluorescent assay, which showed that stachydrine blocked the AngII-induced increased colocalization of gp91phox and p47phox (Fig. 3C). Considering that NOX2 cytosolic subunit translocation requires the phosphorylation of p47phox, we evaluated the phosphorylation level of p47phox. As shown in Fig. 3D, AngII treatment led to enhanced phosphorylation of p47phox, which was abrogated by stachydrine.

\section{Stachydrine alleviated TAC-induced cardiac hypertrophy in rats}

We used the TAC animal model to determine whether stachydrine can alleviate pressure overload-induced cardiac hypertrophy. As shown in Fig. 4B, stachydrine partially but significantly reduced the TAC-induced increase in the heart weight-to-body weight ratio and heart weight-to-tibial length ratio. Meanwhile, telmisartan significantly reduced the increase in the heart weight-to-body weight ratio and completely blocked the increase in the weight-to-tibial length ratio. Stachydrine and telmisartan significantly reduced the TACinduced thickening in the myocardial wall in the diastolic phase, including the left ventricular posterior wall (LVPWd) and inter ventricular septum (IVSd). Stachydrine and telmisartan 


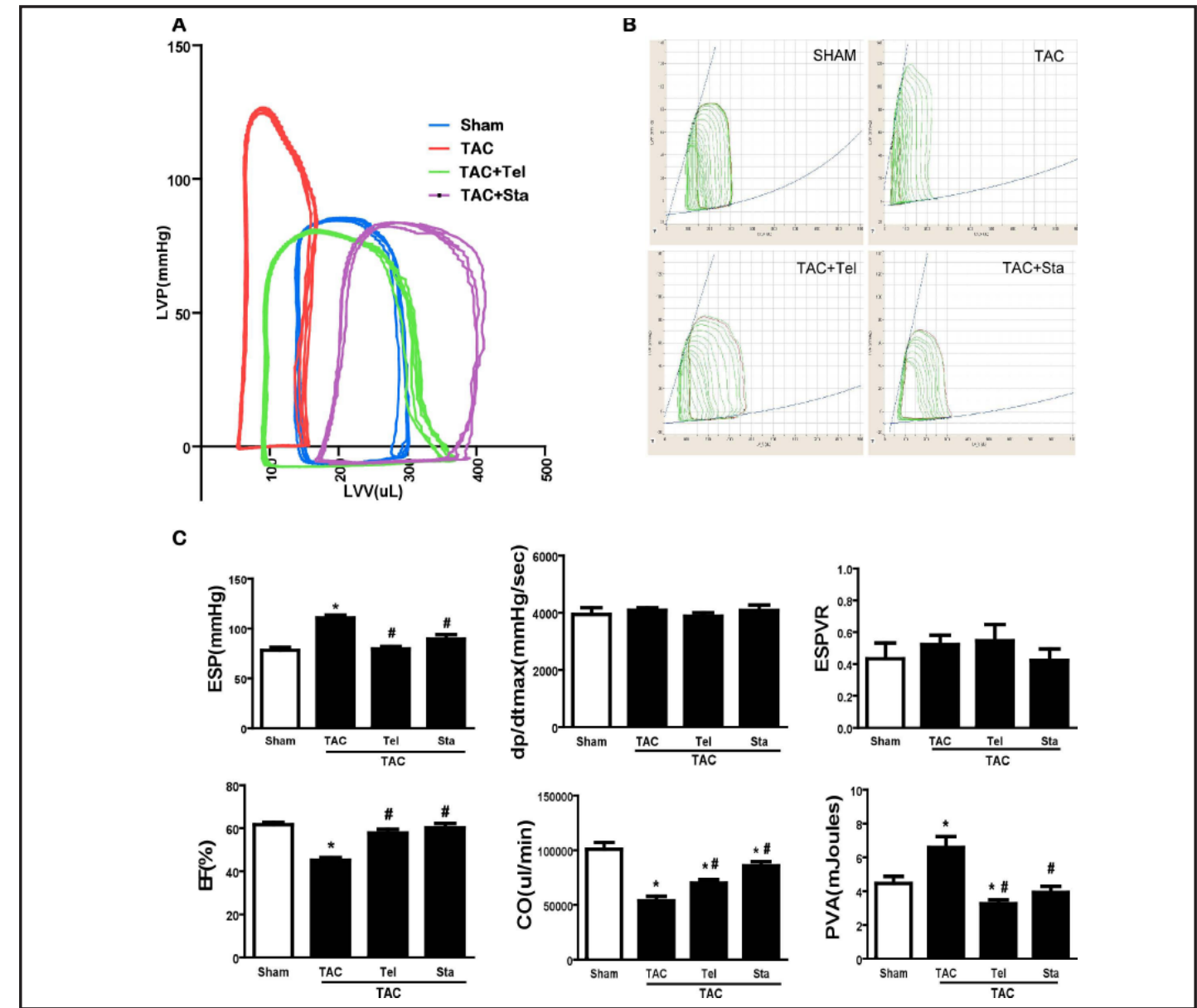

B

Fig. 5. Stachydrine ameliorated TAC-induced cardiac dysfunction in rats. (A) Representative steady-state pressure-volume loops. (B) Representative pressure-volume loops during vena cava occlusion. (C) Quantification of hemodynamic parameters. The data are expressed as the mean \pm SEM. $* \mathrm{P}<0.05$ compared to Sham; \# P<0.05 compared to TAC. Sta, stachydrine; TAC, transverse aorta constriction; Tel, telmisartan.

completely blocked the TAC-induced thickening in the myocardial wall in the systolic phase, including the LVPWs and IVSs (Fig. 4C and 4D). These parameters were consistent with the HE-stained histology sections that showed that stachydrine and telmisartan reduced the thickening of the left ventricular wall induced by TAC (Fig. 4A). Together, the data suggest that stachydrine ameliorated pressure overload-induced myocardial hypertrophy. However, TAC did not alter the left-ventricular internal dimension during diastole and systole (LVIDd and LVIDs), which indicated that, at this point, the animals were still in the compensated hypertrophy period.

\section{Stachydrine ameliorated TAC-induced cardiac dysfunction in rats}

To further gain insight into the effect of stachydrine, we evaluated the cardiac function using a pressure-volume loop analysis. As shown in the Fig. 5, the TAC operation increased the end-systolic pressure (ESP) without altering the maximum time derivative of pressure development (dp/dt max) and the slope of the end-systolic pressure-volume relationship (ESPVR). At the same time, TAC decreased the cardiac output (CO) and left ventricular ejection fractions (EF). Stachydrine restored the values of the ESP, CO and EF. Meanwhile, telmisartan normalized the values of the ESP and EF but not the value of the CO. Moreover, TAC dramatically increased the pressure-volume area (PVA), which was abrogated by stachydrine and telmisartan. These data suggest that the enhanced myocardial oxygen consumption (MVO2) caused by TAC was prevented by stachydrine and telmisartan. 
A
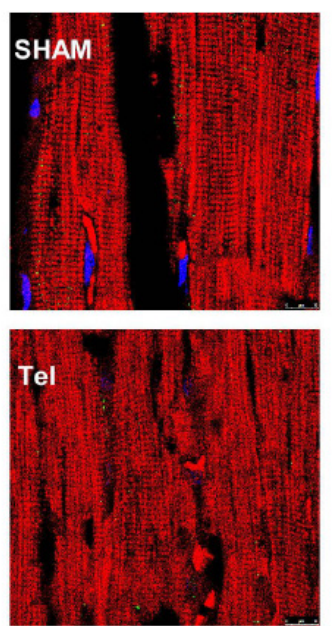

c
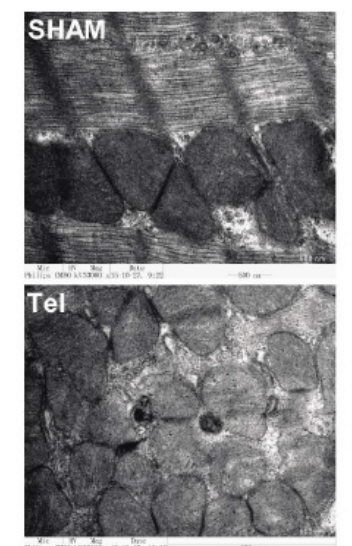
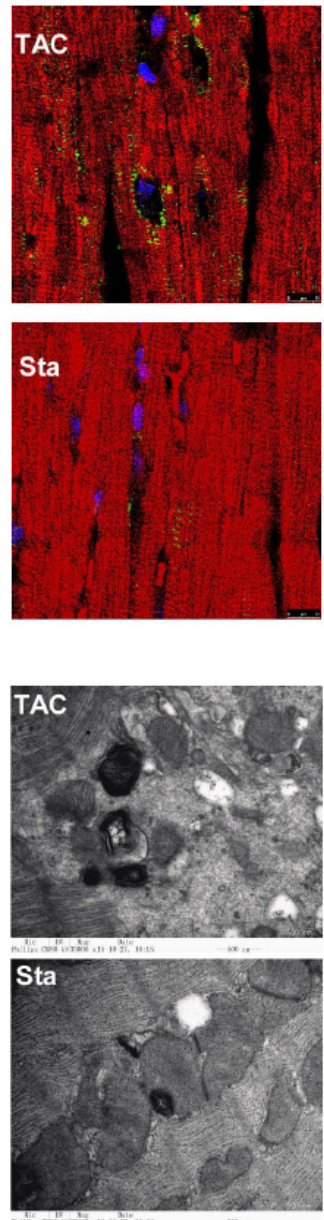

B

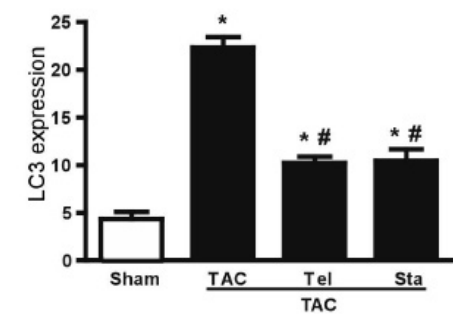

D
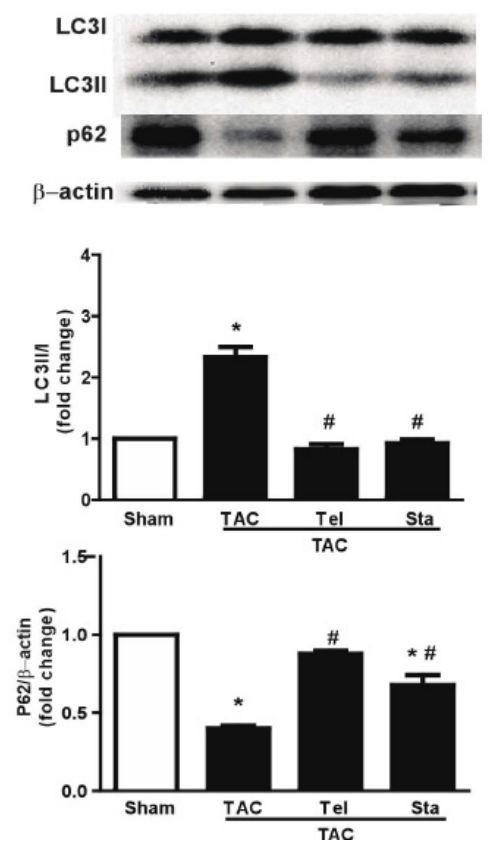

Fig. 6. Stachydrine abrogated TAC-induced excessive autophagic activity in the myocardium in rats. (A) Representative immunofluorescence images with anti-LC3 (green) and anti-cTNT (red), ×400. (B) Quantification of the LC3-positive cell number. (C) Representative electron micrograph shows autophagic vacuoles. (D) SDS-PAGE and western blotting analysis of the LC3II/I ratio and p62 expression. The data are expressed as the mean \pm SEM. ${ }^{*} \mathrm{P}<0.05$ compared to Sham; \# $\mathrm{P}<0.05$ compared to TAC. Sta, stachydrine; TAC, transverse aorta constriction; Tel, telmisartan.

Stachydrine abrogated the TAC-induced excessive autophagic activity in the myocardium in rats.

We next determined whether the downregulation effect of stachydrine on autophagy in vitro could be seen in vivo in rats subjected to the TAC operation. A characteristic of autophagy is the recruitment of microtubule-associated protein 1 light chain 3 (LC3) to autophagic vesicles. Microscopy images showed that stachydrine prevented the TAC-induced increase in the number of LC3 punctate (Fig. 6A, 6B). This is consistent with the western blot analysis that showed that stachydrine abrogated the TAC-induced enhanced LC3-II/LC3-I ratio (Fig. 6D). Furthermore, we also determined the amount of autophagy cargo p62 that was delivered to lysosomes for degradation. We observed a dramatically enhanced degradation rate of p62 in those rats subjected to TAC, which was prevented by stachydrine (Fig. 6D). These data indicate that stachydrine abrogated the pressure overload-induced increase in autophagic activity in vivo. 


\section{Cellular Physiology and Biochemistry Published online: May 12, $2017 \quad \begin{aligned} & \text { Do } 2017 \text { The Author(s). Published by S. Karger AG, Basel } \\ & \text { www.karger.com/cpb }\end{aligned}$

\section{Discussion}

In this study, we demonstrated that stachydrine inhibited excessive autophagic activity both in vitro and in vivo. We found that stachydrine abrogated AngII-induced ROS overproduction by NOX2. We also demonstrated that stachydrine attenuated TAC-induced cardiac hypertrophy and ameliorated cardiac function.

The survival and function of cardiomyocytes critically rely on the basal level of autophagy. Nakai et al. reported that cardiomyocytes manifest a classical hypertrophic response with a reduced autophagic activity [11], while Kanamori et al. reported that during nutrient deprivation, suppressed autophagy leads to reduced cardiac ATP content and impaired heart performance [12]. Furthermore, studies with human samples and pressure overload animal models have suggested that excessive autophagy activity may play a maladaptive role in heart failure pathogenesis [13-17]. In the present study, we found excessive autophagy activity in the myocardium of animals subjected to the TAC operation, correlated with myocardium hypertrophy and cardiac dysfunction. Stachydrine as well as the AngII receptor type I blocker telmisartan inhibited the autophagic activity, attenuated myocardium hypertrophy and ameliorated cardiac function. These data suggested that the inhibition of autophagic activity by stachydrine may play a positive role in its therapeutic effect on pressure overload-induced heart failure.

Accumulating lines of evidence have demonstrated that oxidative stress significantly modulates autophagy during cellular stress [18]. In 2009, Ju Huang found that NOX2 mediated autophagic activity in phagocytes [19]. After that, emerging data have indicated that NOX is involved in the positive regulation of autophagy in several different cell types. Both in vitro and in vivo studies have shown that AngII treatment can lead to an increase in ROS production in the heart, which is significantly inhibited by the NOX inhibitor DPI, suggesting that NOX is the major source of AngII-induced ROS in cardiac myocytes [20,21].

AngII has been shown to increase the expression of NOX2 subunits p22phox, p47phox, p67phox and gp91phox, as well as the translocation of $\mathrm{p} 47$ phox to the membrane, which is consistent with the results of the present study [3]. All of those data suggest that NOX2 is one of the most important sources of AngII-induced ROS production in cardiomyocytes. In the present study, we found that stachydrine inhibited the over-expression of gp91phox and p67phox and blocked p47phox over-phosphorylation and translocation to membrane, thereby inhibiting the excessive activity of NOX2 induced by AngII. These findings are correlated with the negative regulation of autophagy by stachydrine.

\section{Conclusion}

In conclusion, these data suggest that stachydrine suppresses the excessive autophagy activity induced by pressure overload, attenuates myocardial hypertrophy, and improves cardiac performance.

\section{Acknowledgments}

This work was supported by grants from the National Natural Science Foundation of China (30973821; 81373858).

\section{Disclosure Statement}

The authors declare no competing financial interests. 


\section{Cellular Physiology Cell Physiol Biochem 2017;42:103-114 \begin{tabular}{l|l} 
DOI: 10.1159/000477119 & $\begin{array}{l}\text { O 2017 The Author(s). Published by S. Karger AG, Basel } \\
\text { www.karger.com/cpb }\end{array}$ \\
\hline
\end{tabular}

\section{References}

1 Tham YK, Bernardo BC, Ooi JY, Weeks KL, McMullen JR: Pathophysiology of cardiac hypertrophy and heart failure: Signaling pathways and novel therapeutic targets. Arch Toxicol 2015;89:1401-1438.

2 Zhu H, Tannous P, Johnstone JL, Kong Y, Shelton JM, Richardson JA, Le V, Levine B, Rothermel BA, Hill JA: Cardiac autophagy is a maladaptive response to hemodynamic stress. J Clin Invest 2007;117:1782-1793.

- Zablocki D, Sadoshima J: Angiotensin ii and oxidative stress in the failing heart. Antioxid Redox Signal 2013;19:1095-1109.

4 Liu XH, Xin H, Zhu YZ: More than a "mother-benefiting“ herb: Cardioprotective effect of herba leonuri. Sheng li xue bao 2007;59:578-584.

-5 Zhang C, Shan XL, Liao YL, Zhao P, Guo W, Wei HC, Lu R: Effects of stachydrine on norepinephrine-induced neonatal rat cardiac myocytes hypertrophy and intracellular calcium transients. BMC Complement Altern Med. 2014;14:474.

6 Guo W, Zhang C, Liao YL, Lv R, Wei HC: [effect of leonurus stachydrine on myocardial cell hypertrophy]. Zhong Yao Cai. 2012;35:940-943.

-7 Klionsky DJ, Abdelmohsen K, Abe A, Abedin MJ: Guidelines for the use and interpretation of assays for monitoring autophagy (3rd edition). Autophagy 2016;12:1-222.

8 Prosser BL, Ward CW, Lederer WJ: X-ros signaling: Rapid mechano-chemo transduction in heart. Science (New York, NY) 2011;333:1440-1445.

-9 Gottlieb RA, Andres AM, Sin J, Taylor DP: Untangling autophagy measurements: All fluxed up. Circ Res 2015;116:504-514.

10 Burgoyne JR, Mongue-Din H, Eaton P, Shah AM: Redox signaling in cardiac physiology and pathology. Circ Res 2012;111:1091-1106.

-11 Nakai A, Yamaguchi O, Takeda T, Higuchi Y, Hikoso S, Taniike M, Omiya S, Mizote I, Matsumura Y, Asahi M, Nishida K, Hori M, Mizushima N, Otsu K: The role of autophagy in cardiomyocytes in the basal state and in response to hemodynamic stress. Nat Med 2007;13:619-624.

12 Kanamori H, Takemura G, Maruyama R, Goto K, Tsujimoto A, Ogino A, Li L, Kawamura I, Takeyama T, Kawaguchi T, Nagashima K, Fujiwara T, Fujiwara H, Seishima M, Minatoguchi S: Functional significance and morphological characterization of starvation-induced autophagy in the adult heart. Am J Pathol 2009;174:1705-1714.

13 Cao DJ, Wang ZV, Battiprolu PK, Jiang N, Morales CR, Kong Y, Rothermel BA, Gillette TG, Hill JA: Histone deacetylase (hdac) inhibitors attenuate cardiac hypertrophy by suppressing autophagy. Proc Natl Acad Sci U S A 2011;108:4123-4128.

14 Rifki OF, Bodemann BO, Battiprolu PK, White MA, Hill JA: Ralgds-dependent cardiomyocyte autophagy is required for load-induced ventricular hypertrophy. J Mol Cell Cardiol 2013;59:128-138.

15 Kostin S, Pool L, Elsasser A, Hein S, Drexler HC, Arnon E, Hayakawa Y, Zimmermann R, Bauer E, Klovekorn WP, Schaper J: Myocytes die by multiple mechanisms in failing human hearts. Circ Res 2003;92:715-724.

-16 Saijo M, Takemura G, Koda M, Okada H, Miyata S, Ohno Y, Kawasaki M, Tsuchiya K, Nishigaki K, Minatoguchi S, Goto K, Fujiwara H: Cardiomyopathy with prominent autophagic degeneration, accompanied by an elevated plasma brain natriuretic peptide level despite the lack of overt heart failure. Intern Med (Tokyo, Japan) 2004;43:700-703.

17 Liu L, Wang C, Sun D, Jiang S, Li H, Zhang W, Zhao Y, Xi Y, Shi S, Lu F, Tian Y, Xu C, Wang L: Calhex(2)(3)(1) ameliorates cardiac hypertrophy by inhibiting cellular autophagy in vivo and in vitro. Cell Physiol Biochem 2015;36:1597-1612.

18 Hsieh DJ, Kuo WW, Lai YP, Shibu MA, Shen CY, Pai P, Yeh YL, Lin JY, Viswanadha VP, Huang CY: 17betaestradiol and/or estrogen receptor beta attenuate the autophagic and apoptotic effects induced by prolonged hypoxia through hif-1alpha-mediated bnip3 and igfbp-3 signaling blockage. Cell Physiol Biochem 2015;36:274-284.

19 Huang J, Canadien V, Lam GY, Steinberg BE, Dinauer MC, Magalhaes MA, Glogauer M, Grinstein S, Brumell JH: Activation of antibacterial autophagy by nadph oxidases. Proc Natl Acad Sci U S A 2009;106:6226-6231.

20 Vernon PJ, Tang D: Eat-me: Autophagy, phagocytosis, and reactive oxygen species signaling. Antioxid Redox Signal 2013;18:677-691.

21 Kimura S, Noda T, Yoshimori T: Dissection of the autophagosome maturation process by a novel reporter protein, tandem fluorescent-tagged lc3. Autophagy 2007;3:452-460. 